\title{
Olig1 and Olig2 promote oligodendrocyte differentiation of neural stem cells in adult mice injured by EAE
}

\author{
Tamara L. Adams, Joseph M. Verdi*
}

Maine Medical Center Research Institute, 81 Research Drive, Scarborough, USA

Email: ${ }^{*}$ verdij@mmc.org

Received 2 June 2012; revised 28 July 2012; accepted 28 August 2012

\begin{abstract}
Investigating neural stem cell plasticity in the hippocampal niche, we demonstrate that retroviral forced expression of Mash1 (Mammalian Achaete-Scute Homolog 1), Olig1 (Oligodendrocyte transcription factor 1), and Olig2 (Oligodendrocyte transcription factor 2) genes, transcription factors involved in enhanced oligodendrogenesis, can contribute to directing the differentiation of adult subventricular zone neural stem cells to functional oligodendrocytes. We found that Mash1, Olig1 and Olig2 all induced oligodendrocyte differentiation. However, Olig1 and Olig2 induction resulted in an elevated number of generated oligodendrocytes without a significant production of other cell lineages, unlike Mash1. These newly differentiated cells are also capable of migration and possible myelination, showing that targeting oligodendrocyte production and possible remyelination is a viable therapeutic strategy for restoration of neuronal function.
\end{abstract}

Keywords: Mash1; Olig1; Olig2; Oligodendrocyte; NSC; EAE/MS

\section{INTRODUCTION}

Identifying signaling and pathways that govern oligodendrocyte production would allow for the generation of a surplus of oligodendrocyte cells that are impervious from immune rejection and could be used as a therapeutic measure in diseases such as Multiple Sclerosis (MS) where myelination is compromised. In order to ensure the generation of sufficient number of oligodendrocytes needed for effective cell therapy, it would be advantageous to learn how these cells are derived from redirected granule cell precursors and whether induction of transcription factors involved in oligodendrocyte differentiation would improve the number of differentiated oligodendrocytes ([1] Raff et al., 1998, [2] Raff et al., 2001, [3] Stemple et al., 1992).

${ }^{*}$ Corresponding author.
In this study, we investigated neural stem cell plasticity in the hippocampal niche resulting in neuronal repair using a retroviral induction approach of multiple transcription factors to direct NSCs differentiation into oligodendrocytes in an inflammatory microenvironment analogous to that in MS [4] (Navikas et al., 1996). Our intent was to produce a sizeable number of oligodendrocytes from mouse hippocampal neural stem cells in Experimental Autoimmune Encephalomyelitis (EAE) disease model, widely used to study pathogenesis of autoimmunity, demyelination, cell trafficking, and tolerance induction [5] (Olsson et al., 1995).

\section{MATERIALS AND METHODS}

\subsection{Cell Culture}

Forebrains were removed from15-day old mouse fetuses, mechanically dissociated then digested in $0.05 \%$ trypsin (Mediatech, Manassas, VA) for $15 \mathrm{~min}$ at $37^{\circ}$, then washed with $1 \times$ DPBS (Mediatech, Manassas, VA) and passed gently through a $70 \mu \mathrm{m}$ mesh. The cells were then centrifuged then the pellet was resuspended in DMEM/ $10 \% \mathrm{FBS} / 0.28 \%$ BSA. $14 \mathrm{~mm}$ diameter glass coverslips were coated with poly L-lysine (Sigma, St. Louis, MO) and placed in 24 well plates (CellstarOne, Monroe, North Carolina). The cells were plated at $5 \times 104$ cells per coverslip in $10 \mu \mathrm{l}$ of media then placed at $37^{\circ}$ with $5 \% \mathrm{CO}_{2}$ for 30 minutes to allow attachment. $500 \mu \mathrm{l}$ of culture medium was added to each well for 2 weeks, media was replaced twice a week [6] (C. Lubetzki et al., 1993).

\subsection{Generation of EAE Mice}

C57B/6, both male and female, age 10 weeks old, were purchased from Taconic (Hudson, NY) and acclimated to our vivarium. EAE was induced via injections of a commercially available kit from Hooke's Laboratories (San Diego, CA, kit \# EK-0115 (MOG 35-55/CFA Emulsion PTX). This was used according to manufactures protocol. The mice were monitored daily for changes (trembling, limping, partial or full paralysis, and other signs of EAE 
induction) and were scored using Hooke's scale of 0 - 5. Using this system we were able to induce EAE as defined by 24 -hour partial paralysis in greater than $70 \%$ of the mice within 14 days of the final injection of pertussis toxin. All mouse studies were done with IACUC approval with minimal discomfort to animals.

\subsection{Retroviral Production}

The GFP, Mash1, Shh, and Olig1 and Olig2 virus particles were made by cloning each gene into the AP2-GFP retrovirus plasmid via the Xho1 cut site. 293-GPG cells, grown in DMEM media with $10 \%$ FBS and $200 \mathrm{ug} / \mathrm{ml}$ G418, were used to obtain the retrovirus conditioned medium. These cells were transfected with each retrovirus construct using Invitrogen Lipofectamine 2000 Transfection Reagent (Invitrogen, Carlsbad, CA). The conditioned medium was taken every 24 hours. The medium was then concentrated using the ViraBind Retrovirus Concentration and Purification Kit Item \#VPK-131 (Cell Biolabs Inc., San Diego, CA) as protocol required to obtain concentrated virus at $<10$ '5 CFU.

\subsection{Stereotactic Virus Injections}

Mice were injected with Avertin at a concentration of $250 \mathrm{mg} / \mathrm{kg}$ to anesthetize the mice. Then each mouse was stereotactically injected using a Lab Standard Stereotaxic Instrument with Stoelting manual Stereotaxic injector (Stoelting, Wood Dale, IL) at -2 anterior/posterior +1.5 medial/laeral and -2.3 dorsal/ventral from the skull with concentrated virus.

\subsection{Histological Preparation of Sections}

Mice were then euthanized via a $\mathrm{CO}_{2}$ chamber and brains and spinal cord taken for sectioning. Tissue was fixed in $4 \%$ paraformaldehyde prior to sectioning. The specimens were processed using a Leica TP1020 Automated Tissue Processor and a Fisher HistoCenter embedding station, then sectioned at $5 \mu \mathrm{m}$ with a Microm microtome.

\subsection{Immunohistochemistry}

Histological and cell culture sections were performed as previously described in our lab [6] (Matluk et al., 2010). The primary antibodies used: Mouse anti Olig (RIP, Developmental Studies Hybridoma Bank, University of Iowa), Mouse anti-MOG (\#MAB5680, Chemicon of Millipore, Billerica, MA), Mouse anti GFAP (Glial fibrillary acidic protein, \#MAB360, Chemicon of Millipore, Billerica, MA), Rabbit anti Neurofilament (\#041032, Chemicon of Millipore, Billerica, MA). Secondary antibodies were either Alexafluor546 Goat anti-Rabbit IgG, Alexafluor647 Goat anti-Rabbit IgG or Alexafluor546 Goat anti-Mouse IgG (Molecular probes of Invitrogen,
Carlsbad, CA). Pictures were taken using a Ziess Axiovert 200 inverted microscope.

\section{RESULTS}

\subsection{In Vitro Induction of Oligodendrocyte Differentiation}

We focused on transcription factors that have been shown regulate oligodendrocyte differentiation from NSCs (Figure 1(a)). We performed AP2 [4] (Galipeau et al., 1999) retroviral induction of Mash1 [Mammalian AchaeteScute Homolog 1 ([7-9]], Shh [Sonic Hedgehog [10])], Olig1 [Oligodendrocyte transcription factor $([11,12]$ and Olig2 [Oligodendrocyte transcription factor 2 ([13] Du et al., 2006, [14]), (Figure 1(b)) on isolated mouse neural stem cells in culture to analyze their effects on the oligodendrocyte development using previously established methods [15] (C. Lubetzki et al., 1993). We found that Olig2 had the greatest effect on oligodendrocyte differentiation and these newly generated oligodendrocytes were making an effort to myelinate the axons of neurons in cell culture (Figures 2A-F). We demonstrate that NSCs induced with Olig2 retrovirus (Figure 2A) differentiate into oligodendrocytes (Figure 2B) which migrate toward neurons (Figure 2C) and initiate myelination (Figures 2E and F). We found that both Olig1 and Olig2 induced oligodendrocyte differentiation, however, Olig2 induction resulted in a higher and more consistent average of generated oligodendrocytes (Figure 2G). We did not observe a significant synergistic effect of Olig1 and Olig2 induction (Figure 2G). Mash1 and Shh unlike Olig1 and Olig2 produced a small number of oligodendrocytes when induced alone or in combination (Figure 2G). Interestingly, induction of NSCs with combinations of Mash1 or Shh with Olig1 or Olig2 caused a decrease in oligodendrocyte differentiation as compared to Olig1 or Olig2 alone (Figure 2G).

\subsection{In Vivo Induction of Oligodendrocyte Differentiation}

We examined forced retroviral expression of Mash1, Olig1, or Olig-AP2 in differentiating subventricular zone neural stem cells and its effect on oligodendrocyte fate in an inflammatory enriched microenvironment, we examined $\mathrm{EAE} \mathrm{C} 57 \mathrm{~B} / 6$ mice that underwent concentrated retrovirus particles $\left(<10^{\prime} 5 \mathrm{CFU}\right)$ injections in the subventricular zone, following a previously published protocol ([14] Jessberger et al., 2009). At week four, we examined histological sections of the brain to evaluate retrovirally induced cell differentiation to oligodendrogenic, neuronal, and glial lineages using specific lineage markers. Mash1 differentiate stem cells to oligodendrocytes upon viral induction, but concomitantly we found a proliferation of virally induced neurons and astrocytes 


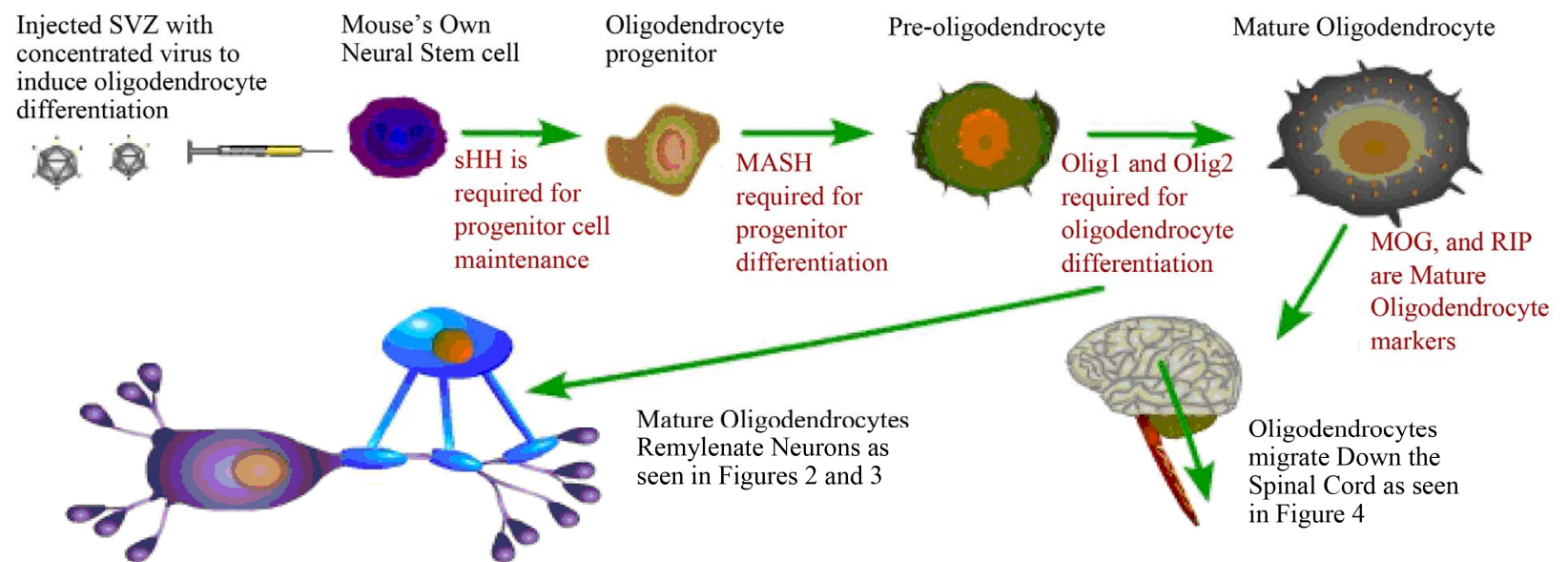

(a)

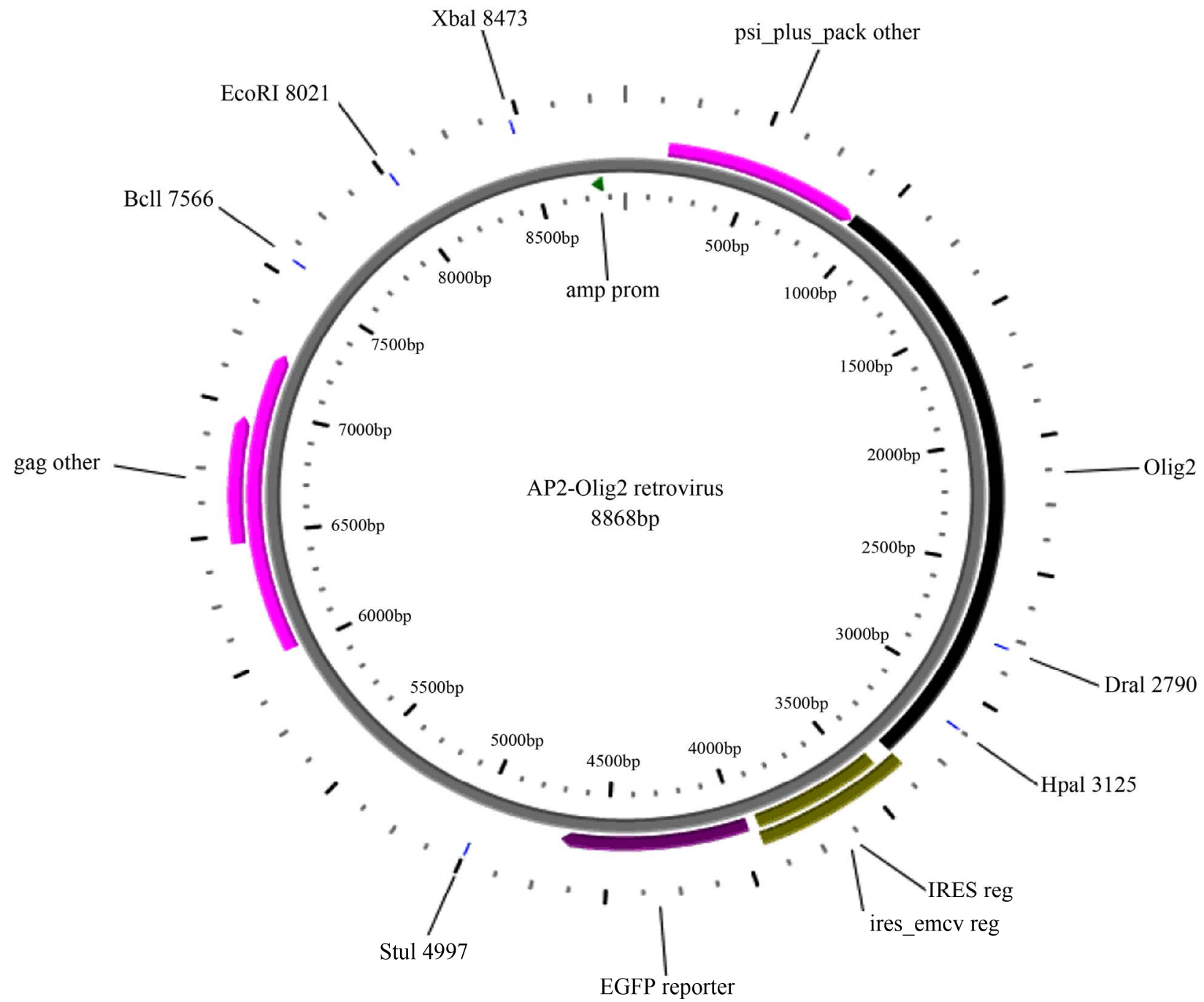

(b)

Figure 1. Schematic outline of forced oligodendrocyte differentiation in EAE induced mice: (a) Our in vivo schematic model depicts forced induction of differentiated subventricular zone neural stem cells to oligodendrocytes in EAE induced adult mice that begin to wrap neurons and migrate down the spinal cord; (b) Plasmid map of Olig2-AP2-GFP retrovirus constructs used in viral transduction. This map is representative of all retroviral constructs used in this study. 

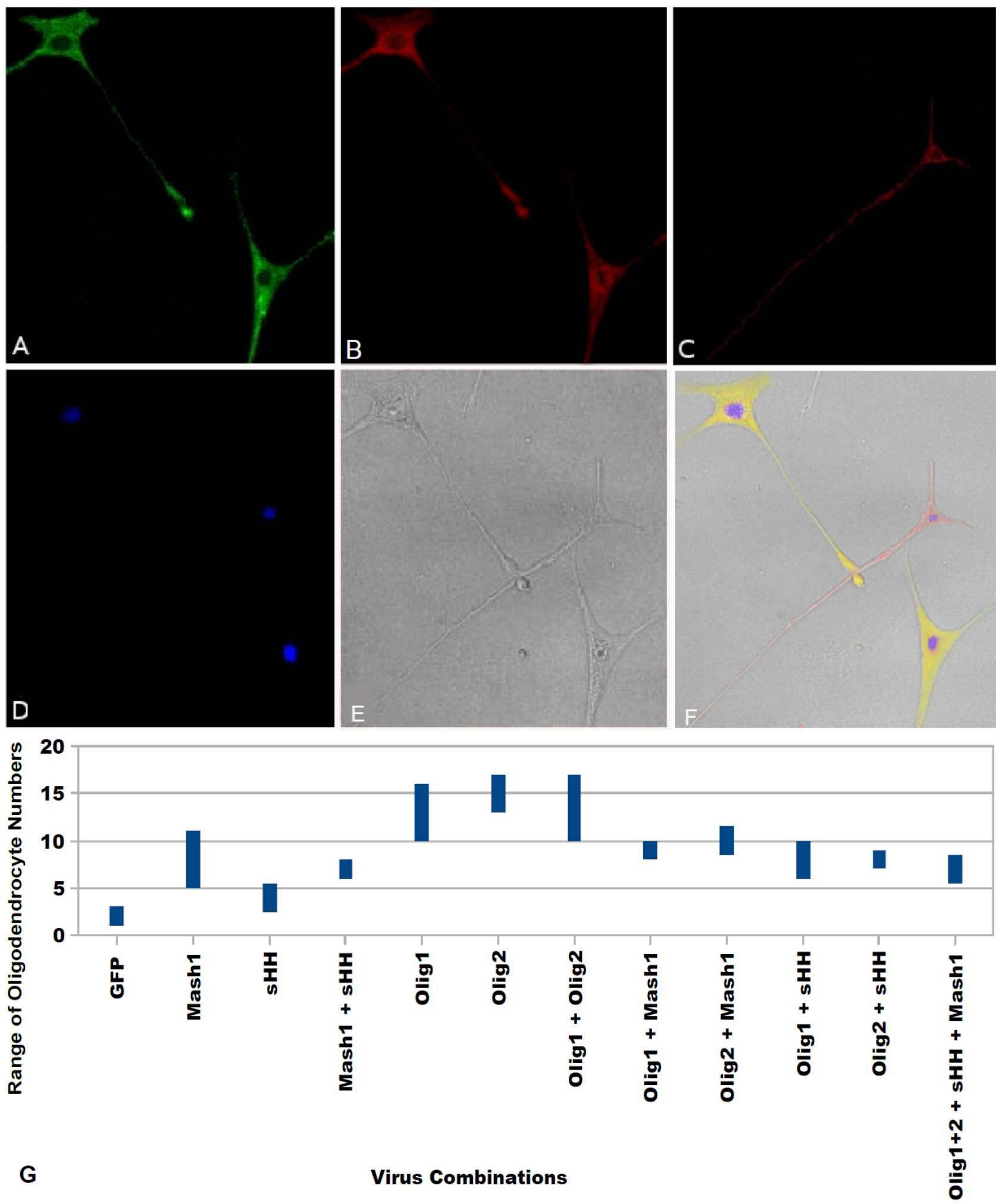

Figure 2. In vitro forced oligodendrocyte differentiation using Olig2 retrovirus. (A) Forced expression of Olig2-AP2-GFP in differentiated oligodendrocytes, Green depicts positive Olig2 retroviral induction, 40×; (B) Positive staining for MBP, a specific marker for oligodendrocyte, 40×; (C) Positive staining for neurofilament depicts neuron in close proximity to oligodendrocyte, 40×; (D) Dapi staining for nuclei; (E) Oligodendrocyte differentiated from SVZ NSCs using Olig2-AP2-GFP retroviral induction, phase contrast, 40×; (F) Overlayed image of (A-E) depicts growth of generated oligodendrocyte that makes contact with neuron and can potentially myelinate the axon of neuron. 

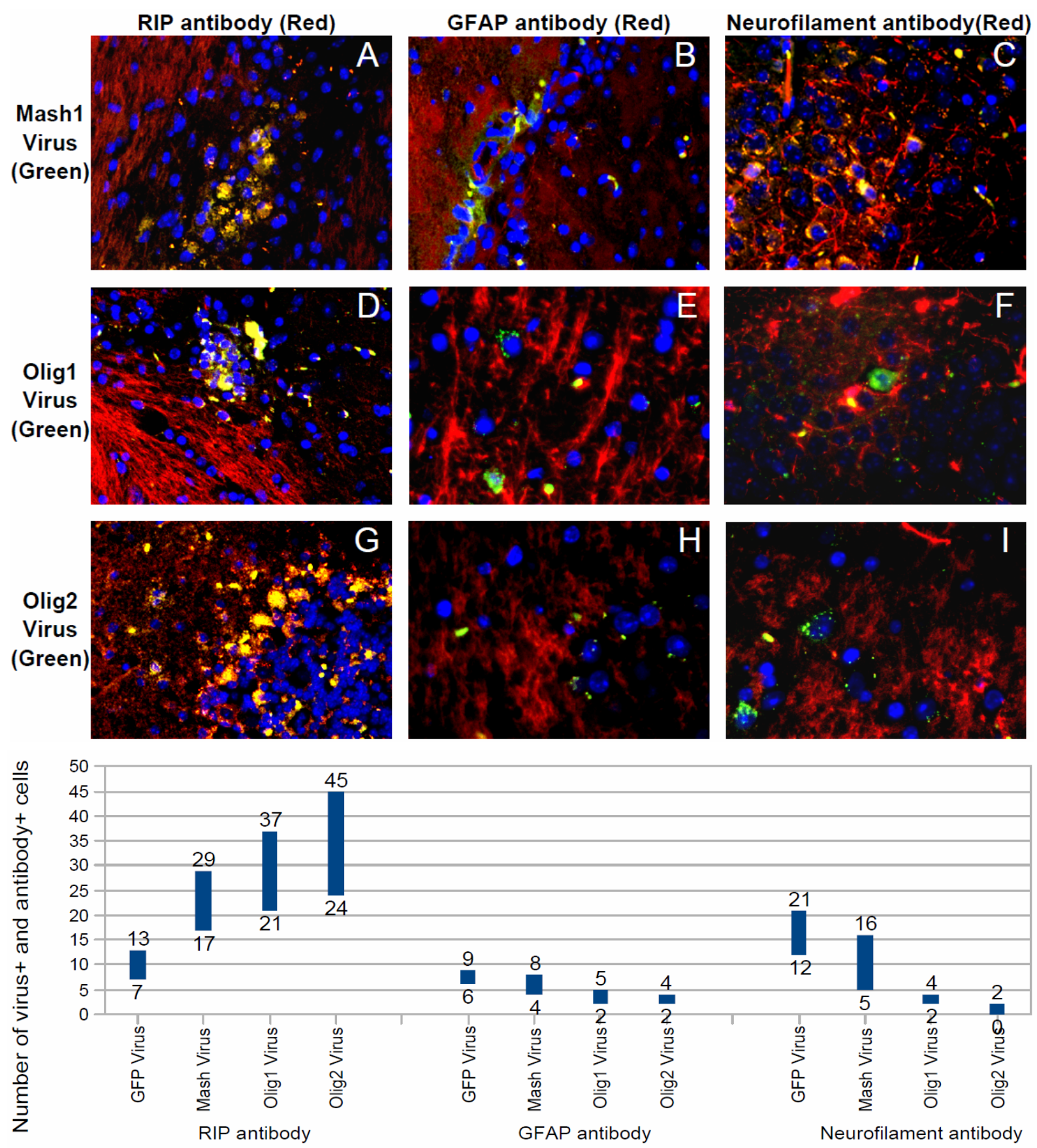

Figure 3. Mash1, Olig1, and Olig2 direct the differentiation of SVZ progenitor cells to oligodendrocytes. (A) Immunohistochemical analysis of Mash1-AP2-GFP positive cells (Green) that was overlapped with staining of RIP, a marker for oligodendrocytes. Yellow depicts co-expression; (B) Mash1-AP2-GFP positive cells (Green) merged with overlap of GFAP, marker for astrocyte lineage (Red). Yellow depicts co-expression; (C) Mash1-AP2-GFP positive cells (Green) merged with overlap of neurofilament, marker for neurons (Red). Yellow depicts co-expression; (D) Immunohistochemical analysis of Olig1-AP2-GFP positive cells (Green) merged with overlap of RIP (Red). Yellow depicts co-expression; (E) Olig1-AP2GFP positive cells (Green) merged with overlap of GFAP (Red). Yellow depicts co-expression; (F) Olig1-AP2-GFP positive cells (Green) merged with overlap of neurofilament (Red). Yellow depicts co-expression; (G) Immunohistochemical analysis of Olig2-AP2-GFP positive cells (Green) merged with overlap of RIP (Red). Yellow depicts co-expression; (H) Olig2AP2-GFP positive cells (Green) merged with overlap of GFAP (Red). Yellow depicts coexpression; (I) Olig2-AP2-GFP positive cells (Green) merged with overlap of neurofilament (Red). Yellow depicts co-expression; (J) Chart illustrating the range of cell numbers found to co-express virus and antibody for each virus ( $\mathrm{N}=12$ for each virus). 

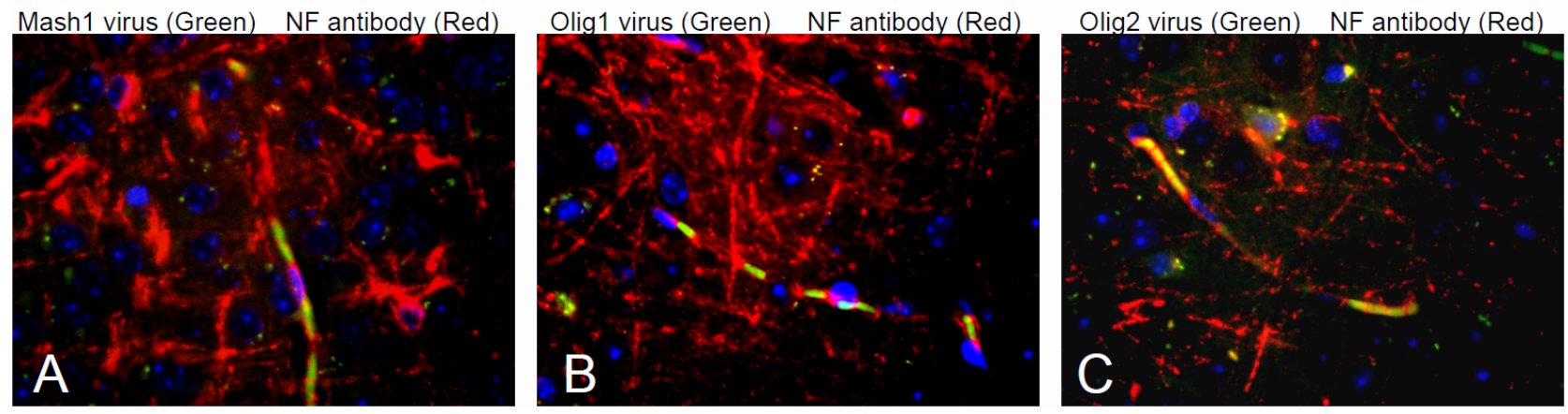

Figure 4. Virus induced oligodendrocytes appear to myelinate neurons in vivo. (A) Mash1 induced oligodendrocytes appear to myelinate local neurons. Mash1-AP2-GFP (Green) Neurofilament (Red); (B) Olig1 induced oligodendrocytes appear to myelinate local neurons. Olig1-AP2-GFP (Green) Neurofilament (Red); (C) Olig2 induced oligodendrocytes appear to myelinate local neurons. Olig2-AP2-GFP (Green) Neurofilament (Red).
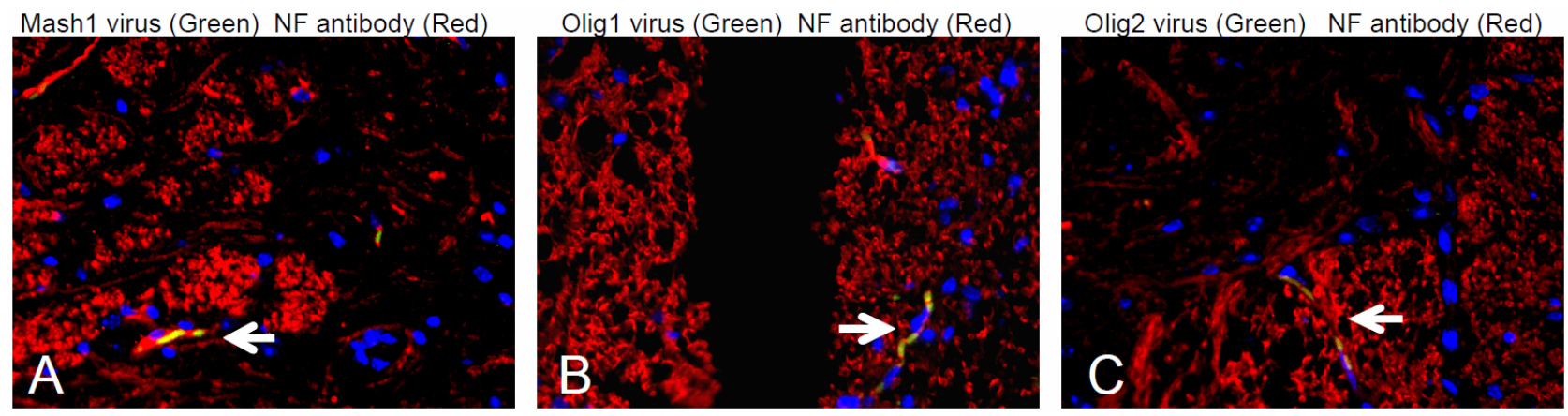

Figure 5. Virus induced oligodendrocytes migrate down the spinal cord. (A) Mash1 induced oligodendrocytes migrate to $2 \mathrm{~cm}$ down the spinal cord. Mash1-AP2-GFP (Green) Neurofilament (Red); (B) Olig1 induced oligodendrocytes migrate to $3 \mathrm{~cm}$ down the spinal cord. Olig1-AP2-GFP (Green), Neurofilament (Red); (C) Olig2 induced oligodendrocytes migrate to $3 \mathrm{~cm}$ down the spinal cord. Olig2-AP2-GFP (Green), Neurofilament (Red).

(Figures 3A-C). Previous work implicates that Olig1 and Olig2 genes have positive effects on the oligodendrocyte differentiation process [16] (Alberta et al., 2001, [15] Du ZW et al., 2006, and [17] Maire CL et al., 2010). Our findings revealed that Olig1 (Figure 3D) and Olig2 (Figure 3G) induction resulted in a significant increase of differentiated oligodendrocytes as compared to Mash1. In contrast to Mash1, cells from neuron and astrocyte lineages were not abundant in either Olig1 or Olig2 induction (Figures 3E, F, H, I). These findings, when compared to the GFP-AP2 virus control, suggest that Olig1 and Olig2 induction results in an increased population of differentiated oligodendrocytes without a significant production of other cell lineages (Figure $3 \mathbf{J}$ ).

\subsection{Oligodendrocytes Derived from SVZ Progenitors Possibly Myelinate and Migrate to Spinal Cord}

We next examined whether differentiated oligodendrocytes from the SVZ in EAE mice would myelinate neurons or migrate within the central nervous system. We observed that Mash1, Olig1, and Olig2-AP2 viral expressing oligodendrocytes were abundant and close to neurons detected with antibody for neurofilament protein NF with the prospective to myelinate (Figures 4A-C). These newly differentiated stem cells show great potential in their ability to function as mature oligodendrocytes in an adult mouse model by making contact with nearby neurons. Using a specific marker for oligodendrocytes (RIP) we investigated these differentiated oligodendrocytes migrating a distance of several centimeters within the central nervous system of EAE induced C56B/6 mice. To show their migrating ability, we analyzed spinal cord sections up to $3 \mathrm{~cm}$ down each spine and found that virus induced differentiated oligodendrocytes were observed to have migrated the entire length of these sections (Figures 5A-C), giving support to the plasticity of adult mouse neural stem cells. These observations along with the significant increase in oligodendrocyte production from SVZ-derived cells in the brain injured by EAE provide additional evidence that predominatly Olig1 and Olig2 expression in SVZ cells directs them to a mature oligodendrocyte fate.

\section{DISCUSSION}

We have demonstrated that retroviral forced expression 
of Mash1, Olig1, and Olig2 genes, involved in enhanced oligodendrogenesis, can contribute to directing the differentiation of adult subventricular zone neural stem cells to functional oligodendrocytes. Mash1 alone or with Shh produced oligodendrocytes, but it was Olig1 and Olig2 that gave us a much larger increase in oligodendrocyte numbers while creating fewer cells from the neuronal lineage. These oligodendrocytes are also capable of migration and possible myelination. These findings could allow us to design small molecules that would be used to force ones own neural stem cells to make oligodendrocytes and remyelinate the damaged axons and consequently restore neuronal function.

These reports show that targeting oligodendrocyte production and possible remylination is a viable therapeutic strategy for restoration of neuronal function. We envision that results of the proposed study may extend to several neurological disorders where cellular replacement is needed. Future work should be done to find whether all the oligodendrocytes formed are mature enough to partake in full remyelination of axons that have loss of function due to EAE.

\section{ACKNOWLEDGEMENTS}

The authors would like to thank Dr. Jennifer Rochira and Dr. Aldona Karaczyn for their knowledge and insight for experimental design. Thank you to Dr. Josephine Nalbantoglu for sharing the AP2 retrovirus construct. T.A. carried out experiments, analyzed data, and wrote the manuscript. J.M.V. conceived the experimental design. Authors have no conflict of interest.

\section{REFERENCES}

[1] Raff, M., Apperly, J., Kondo, T., Tokumoto, Y. and Tang, D. (2001) Timing cell-cycle exit and differentiation in oligodendrocyte development. Novartis Found Symposia, 237, 100-107; discussion 107-112, 158-163.

[2] Raff, M.C., Durand, B. and Gao, F.B. (1998) Cell number control and timing in animal development: The oligodendrocyte cell lineage. International Journal of Development Biology, 42, 263-267.

[3] Stemple, D.L. and Anderson, D.J. (1992) Isolation of a stem cell for neurons and glia from the mammalian neural crest. Cell, 71, 973-985.

doi:10.1016/0092-8674(92)90393-Q

[4] Galipeau, J., Andre, H.L., Paquin, A., Sicilia, F., Karpati, G. and Nalbantoglu, J. (1999) Vasicular stomatitis virus g pseudotyped retrovector mediates effective in vivo suicide gene delivery in experimental brain cancer. Cancer Research, 59, 2384-2394.

[5] Olsson, T. (1995) Critical influences of the cytokine orchestration on the outcome of myelin antigenspecific T-cell autoimmunity in experimental autoimmune encephalomyelitis and multiple sclerosis. Immunological Reviews, 144, 245-268.
doi:10.1111/j.1600-065X.1995.tb00072.X

[6] Matluk, N., Rochira, J., Karaczyn, A., Adams, T. and Verdi, J.M. (2010) A role for NRAGE in NF- $\kappa$ B activation through the non-canonical BMP pathway. BMC Biology, 8, 7. doi:10.1186/1741-7007-8-7

[7] Parras, C.M., Hunt, C., Sugimori, M., Nakafuku, M., Rowitch, D. and Guillemot, F. (2007) The proneural gene Mash1 specifies an early population of telencephalic oligodendrocytes. The Journal of Neuroscience, 27, 42334242. doi:10.1523/JNEUROSCI.0126-07.2007

[8] Johnson, J. E., Birren, S. J., Saito, T. and Anderson, D. J. (1992) DNA binding and transcriptional regulatory activity of mammalian achaete-scute homologous (MASH) proteins revealed by interaction with a muscle-specific enhancer. Proceedings of the National Academy of Sciences, 89, 3596-3600. doi:10.1073/pnas.89.8.3596

[9] Rogister, B., Ben-Hur, T. and Dubois-Dalcq, M. (1999) From neural stem cells to myelinating oligodendrocytes. Molecular and Cellular Neuroscience, 14, 287-300. doi:10.1006/mene.1999.0790

[10] Alberta, J.A., Park, S.K., Mora, J., Yuk, D., Pawlitzky, I., Iannarelli, P., Vartanian, T., Stiles, C.D. and Rowitch, D.H. (2001) Sonic hedgehog is required during an early phase of oligodendrocyte development in mammalian brain. Molecular and Cellular Neuroscience, 18, 434-441. doi:10.1006/mone.2001.1026

[11] Rogister, B., Ben-Hur, T. and Dubois-Dalcq, M. (1999) From neural stem cells to myelinating oligodendrocytes. Molecular and Cellular Neuroscience, 14, 287-300. doi:10.1006/mene.1999.0790

[12] Othman, A., Frim, D.M., Polak, P., Vujicic, S., Arnason, B.G. and Boullerne, A.I. (2011) Olig1 is expressed in human oligodendrocytes during maturation and regeneration. Glia, 59, 914-926. doi:10.1002/glia.21163

[13] Du, Z.W., Li, X.J., Ngyen, G.D. and Zhang, S.C. (2006) Induced expression of Olig2 is sufficient for oligodendrocyte specification but not for motoneuron specification and astrocyte repression. Molecular and Cellular Neuroscience, 33, 371-380. doi:10.1016/j.men.2006.08.007

[14] Jessberger, S. and Gage, F.H. (2009) Fate plasticity of adult hippocampal progenitors: Biological relevance and therapeutic use. Trends in Pharmacological Sciences, 30, 61-65. doi:10.1016/j.tips.2008.11.003

[15] Lubetzki, C., Demerens, C., Anglade, P., Villarroya, A., Frankfurter, A., Lee, M.Y. and Zalc, B. (1993) Even in culture, oligodendrocytes meyelinate solely axons. Proceedings of the National Academy Sciences, 40, 68206824.

[16] Zhu, G., Mehler, M.F., Zhao, J., Yu Yung, S. and Kessler, J.A. (1999) Sonic hedgehog and BMP2 exert opposing actions on proliferation and differentiation of embryonic neural progenitor cells. Developmental Biology, 215, 118129. doi:10.1006/dbio.1999.9431

[17] Maire, C.L., Wegener, A., Kerinon, C. and Nait Oumesmar, B. (2010) Gain-of-function of Olig transcription factors enhances oligodendrogenesis and myelination. Stem Cells, 28, 1611-1622. doi:10.1002/stem. 480 